\title{
Mandibular advancement splints for sleep apnoea
}

The effect of a mandibular advancement splint in subjects with sleep-related breathing disorders

A. Johal, D. Arya, L. J. Winchester, P. J. H. Venn and H. Brooks Br Dent J 2005; 199: 591-596

\section{Objective}

This study assessed the effectiveness of a mandibular advancement splint (MAS) in subjects with sleep-related breathing disorders using both objective and subjective outcome measures.

Design

The study was carried out as a retrospective analysis.

Setting

The study was conducted within the Sleep Studies Unit at the Queen Victoria Hospital, East Grinstead, between May 1997 and March 2000. Subjects and methods

Twenty subjects with obstructive sleep apnoea (OSA) and six with nonapnoeic snoring, diagnosed by overnight polysomnography, were fitted with a monobloc appliance between May 1997 and March 2000.

Main outcome measures

The subjects were analysed for changes in the respiratory disturbance index (RDI) and Epworth Sleepiness Scale (ESS) scores. In addition each subject completed an outcome questionnaire following fitting of the appliance.

Results

Variability in response measured by the change in the respiratory disturbance index was found with no correlation to the baseline recording. Although median RDI values improved in both groups, significantly so in the obstructive sleep apnoea group $(p<0.05)$, seven subjects exhibited an increased RDI score following mandibular advancement splint therapy. The median Epworth Sleepiness Scale scores decreased in both the OSA group and the non-apnoeic snorers although not significantly. Twenty-one of the 26 subjects completed the outcome questionnaire revealing an $81 \%$ reduction in snoring. Sideeffects were generally transient and minor. Eighty-six per cent of the subjects' partners reported better quality of sleep as a result of MAS therapy.

Conclusions

The monobloc appliance significantly improved the Respiratory Disturbance Index in the obstructive sleep apnoea group. Some subjects had increased RDI scores following splint therapy. This supports the need for an objective assessment in the follow-up of patients treated with mandibular advancement splints.

\section{IN BRIEF}

- Mandibular advancement splints can be successfully used in the treatment of patients with sleep-related breathing disorders.

- Patients presenting with obstructive sleep apnoea can have a wide range of disease severity, which may not be indicative of treatment outcome.

- Follow-up sleep studies are required.

\section{COMMENT}

The management of snoring and obstructive sleep apnoea (OSA) now frequently includes the use of mandibular advancement splints (MAS). Until relatively recently these were usually provided by hospital or university-based orthodontists in association with dedicated sleep clinics. Several key studies within the last 10 years have demonstrated the effectiveness of these appliances, and an increasing number of general dental practitioners are now providing MAS appliances for their patients.

This interesting paper reports the use of a one-piece acrylic MAS in a mixed sample of six patients with non-apnoeic snoring and 20 patients with OSA. The overall success rate in reducing the loudness of snoring and improving the quality of bed partners' sleep was more than $80 \%$ which is similar to that found in previous studies. Within the OSA subgroup there was also a significant reduction in the severity of apnoeic nocturnal breathing disturbance as measured using the respiratory disturbance index (RDI).

The paper highlights some important issues in the management of patients with OSA and snoring. In particular, more than a quarter of the patients exhibited a worsening of their RDI when wearing their MAS appliances. Similar findings have been reported in other studies of MAS treatment of OSA and indicate that follow-up sleep studies for MAS-treated patients are essential, particularly in view of the increasing body of evidence indicating an association of OSA with cardiovascular problems. The finding that the response to treatment was unpredictable and was not related to the initial severity of OSA further supports this need for follow-up sleep studies.

Dentists managing patients with snoring and OSA should ensure that those snorers with associated OSA are identified and that OSA patients have adequate follow-up to identify those who have either not improved with treatment or whose OSA may have increased in severity. Patients who do not respond favourably may often benefit from other treatment options including continuous positive airway pressure for OSA, or pharyngeal surgery for non-apnoeic snoring.

\section{Johnston, Consultant Orthodontist and Senior Lecturer, Belfast} School of Dentistry doi: 10.1038/sj.bdj.4812904 